Preprints of the

Max Planck Institute for

Research on Collective Goods

Bonn 2017/13

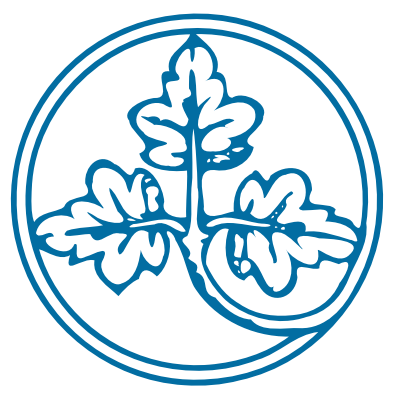

Strategy-proofness of stochastic assignment mechanisms

André Schmelzer

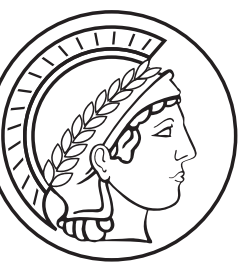




\section{Strategy-proofness of stochastic assignment mechanisms}

André Schmelzer

July 2017 


\title{
Strategy-proofness of stochastic assignment mechanisms
}

\author{
André Schmelzer*
}

June 2017

\begin{abstract}
This paper compares two prominent stochastic assignment mechanisms in the laboratory: Random serial dictatorship (RSD) and top trading cycles with random endowments (TTC). In standard theory, both mechanisms are strategy-proof and Pareto-efficient for the house allocation problem without endowments. In the experiment, RSD outperforms TTC. This can be attributed to more dominant strategy play under RSD. The behavioral theory of obvious strategy-proofness can partly explain this difference in dominant strategy play. Generally, subjects with extremely high and low levels of contingent reasoning play their dominant strategies. These results suggest that one strategy-proof mechanism may outperform another one if individuals are boundedly rational.
\end{abstract}

Keywords: market design, mechanism design, randomization.

${ }^{*}$ Max Planck Institute for Research on Collective Goods, Kurt-Schumacher-Str. 10, D-53113 Bonn, Germany. email: schmelzer@coll.mpg.de.

I thank Christoph Engel, Rustamdjan Hakimov, Yoan Hermstrüwer, Svenja Hippel, Dorothea Kübler, and seminar participants at the MPI for Collective Goods and HU Berlin for helpful discussions and suggestions. Financial support from the Max Planck Society for the Advancement of Science is gratefully acknowledged. 


\section{Introduction}

In principle, stochastic assignment mechanisms are regarded as fair because chance treats everyone equally. However, these mechanisms may not yield the optimal result if individuals do not truthfully reveal their preferences. What may be worse: Individuals who do not understand the dominant strategy could be disadvantaged. In this paper, I study market designs for the stochastic assignment of $n$ indivisible objects to $n$ agents without existing endowments or monetary transfers. Two prominent one-sided matching mechanisms are compared in the laboratory: Random serial dictatorship (RSD) and Gale's top trading cycles algorithm (Shapley and Scarf, 1974) with random endowments (TTC). In RSD, agents are randomly ordered and then objects are assigned in this order according to their preference. In TTC, initially objects are randomly allocated to agents. Then, mutually beneficial exchanges are performed based on agents' preferences. Both mechanisms are strategy-proof and ex-post Pareto-efficient (Abdulkadiroğlu and Sönmez, 1998). In standard theory, they are equivalent.

In practice, it is less clear whether this equivalence holds with boundedly rational agents. Recently, Moraga and Rapoport (2014) proposed to implement TTC and RSD for refugee resettlement. Accordingly, indivisible goods (residence permits) are assigned to agents (refugees) without endowments (pre-existing right to enter a country). Other applications include time slots to users of a common machine, night shifts to doctors, or public housing to tenants. In such real life problems, understandability is key since the efficiency of the outcome depends on the agents' ability to comprehend the dominant strategy. ${ }^{1}$ The planners' choice between theoretically identical mechanisms matters if the dominant strategy is easier to recognize under one mechanism than under the other. Up to now, there is no evidence which allows a comparison of TTC and RSD without endowments.

Assuming bounded rationality, the theory of obvious strategy-proofness (OSP) (Li, 2016) predicts differences in dominant strategy play between RSD and TTC. A mechanism is obviously strategy-proof if recognizing the dominant strategy does not require contingent reasoning about hypothetical actions of others. The se-

\footnotetext{
${ }^{1}$ In the field, failures to recognize dominant strategies are partly found to be strategically motivated (Rees-Jones, 2017) and persist even if information about the dominant strategy is provided (Hassidim et al., 2016).
} 
quential version of RSD (OSP-RSD) is obviously strategy-proof because agents are taking turns and choose an object from a set, one after another. They play the dominant strategy by picking the highest prize. When making their choice, they do not need to reason contingently about the hypothetical choices of the others to play the dominant strategy. In contrast, TTC and the simultaneous version of RSD (SP-RSD) are not obviously strategy-proof because playing the dominant strategy requires contingent reasoning. To recognize the dominant strategy when submitting their rank order list to the mechanisms, the agents need to think about the hypothetical lists of the other agents contingent on their own list. Thus, OSP predicts for boundedly rational agents who do not perfectly reason contingently that the frequency of dominant strategy play is larger in OSP-RSD compared to TTC and SP-RSD.

In this paper, I test the performance of TTC and RSD in the laboratory. I investigate whether dominant strategy play differs between mechanisms and whether the Pareto-efficient outcome is attained. The experiment is designed to compare TTC with both versions of RSD; with the simultaneous SP-RSD and with the sequential, obviously strategy-proof OSP-RSD.

The results clearly show that RSD outperforms TTC. The Pareto-efficient welfare level is attained more often in SP-RSD than in TTC. This can be attributed to more dominant strategy play in the RSD mechanisms compared to TTC. This contrasts standard game theory. As predicted by OSP, there is more dominant strategy play in OSP-RSD than in TTC. However, OSP fails to explain why there is more dominant strategy play in SP-RSD compared to TTC and no differences in dominant strategy play between OSP-RSD and SP-RSD.

These findings complement the matching literature on the house allocation problem with existing endowments, e.g., squatting rights. Abdulkadiroğlu and Sönmez (1999) show that in this case TTC is Pareto-efficient, but RSD is not, because it is not individually rational for every agent to participate in RSD in the first place. Laboratory experiments under incomplete (Chen and Sönmez, 2002) and complete information (Chen and Sönmez, 2004) find that, in line with this theory, RSD is less efficient than TTC. This problem is also known as the housing market with old and new tenants. In contrast, I study the case with new tenants only. This special case is relevant whenever agents do not have prior claims for an 
object like in assigning slots, licenses, and permits.

The closest related paper is Li (2016). He formulates the theory of OSP, taking the limited ability for contingent reasoning into account, and tests it experimentally. $^{2}$ He finds that dominant strategy play and efficiency are larger in sequential RSD than in simultaneous RSD. In addition, my paper directly compares TTC with both versions of RSD. With this comparison, I complement the literature indicating that individuals fail to always play dominant strategies in TTC (e.g., Guillen and Hakimov, 2016) and in RSD (Olson and Porter, 1994).

In the experiment, I introduce a new method to compare sequential and simultaneous mechanisms. My aim is to make decisions more comparable between simultaneously submitting a list of preferences and sequentially selecting an object from a set. I argue that comparing dominant strategy play between a list of 4 preferences with a single choice of an object is not fair because subjects are more likely to make an error when submitting a list. I assume that ranking 4 objects in a list involves making a choice about each individual rank of an object, e.g., by pairwise comparisons. I use the strategy method (Selten, 1967) to implement the sequential OSP-RSD: Subjects choose from 4 different sets of objects without knowing their position in the random sequence. As a result, a preference list containing 4 objects is comparable with 4 choices from sets of objects. It turns out that this method yields different results compared to earlier works. I replicate the finding of Li (2016) that OSP-RSD yields more dominant strategy play than SP-RSD, when I use one single choice from one set of objects using the strategy method data. However, the error rate increases if I use all 4 choices from 4 sets, resulting in the difference between the sequential OSP-RSD and the simultaneous SP-RSD vanishing.

To shed light on the behavioral mechanism behind dominant strategy play, I identify extreme forms of contingent reasoning based on two additional games. At one extreme, I identify subjects who are perfectly able to reason hypothetically about the actions of others by guessing 0 in the 2-person beauty contest game

\footnotetext{
${ }^{2}$ Theory on OSP is evolving. For instance, Pycia and Troyan (2016) introduce a refinement by showing that the class of sequential dictatorships is strong OSP. Troyan (2016) characterizes an OSP implementation of TTC beyond the housing allocation problem. Ashlagi and Gonczarowski (2015) show that the Gale-Shapley deferred acceptance algorithm is not OSP. Zhang and Levin (2017) provide an axiomatization of the failure to reason state-by-state.
} 
(Grosskopf and Nagel, 2008). At the other extreme, I identify subjects who do not engage in hypothetical thinking about the actions of others by requesting 20 in the 11-20 money request game (Arad and Rubinstein, 2012). The upside of this second game: Requesting 20 does not require contingent reasoning, but is at the same time a sensible answer to not knowing what the other person requests. Both extreme types play their dominant strategies. In this way, my work relates to the growing literature on contingent reasoning in other settings such as school choice (Zhang, 2016), takeover games (Charness and Levin, 2009), financial markets (Ngangoue and Weizsäcker, 2015), voting (Esponda and Vespa, 2014), and in explaining the Sure-Thing Principle (Esponda and Vespa, 2016).

The main contributions of my paper are as follows.

(1) RSD outperforms TTC. The theory of obvious strategy-proofness can explain more dominant strategy play in OSP-RSD compared to TTC, but it cannot explain more dominant strategy play in SP-RSD compared to TTC.

(2) OSP-RSD does not outperform SP-RSD. Based on a new method to compare simultaneous with sequential mechanisms, I find that there is no difference in dominant strategy play between the obviously strategy-proof and the strategy-proof version of RSD.

(3) The ability for contingent reasoning predicts dominant strategy play. Based on two additional games, I find that subjects with a perfect ability for contingent reasoning, as well as subjects with no ability for contingent reasoning, play dominant strategies.

(4) Subjects have preferences over mechanisms. A fraction of $40 \%$ of the subjects strictly prefers one mechanism to the other.

The remainder of the paper is organized as follows. Section 2 describes the assignment mechanisms, their theoretical properties, and the resulting predictions for the experiment. The experimental design is described in Section 3. Section 4 provides the main results. Section 5 concludes. 


\section{Theoretical framework and mechanisms}

Consider the house allocation problem as a triple $\langle I, O, \succ\rangle$, where $I$ is a set of agents, $O$ is a set of objects, and $\succ$ are strict preference profiles. Let $|I|=|O|$. An assignment is $\mu: I \rightarrow O$. I rely on the standard formulation of Hylland and Zeckhauser (1979).

Parametrization. In the experiment, four indivisible objects $O=\{a, b, c, d\}$ are assigned to four agents $I=\{1,2,3,4\}$. Each agent is assigned exactly one object. The agents' strict preference profiles $\succ_{i}$ are common knowledge:

\begin{tabular}{c|c|c|c}
$\succ_{1}$ & $\succ_{2}$ & $\succ_{3}$ & $\succ_{4}$ \\
\hline$a$ & $a$ & $a$ & $a$ \\
$b$ & $b$ & $d$ & $d$ \\
$c$ & $c$ & $c$ & $c$ \\
$d$ & $d$ & $b$ & $b$
\end{tabular}

The preference profiles are aligned. Agents form pairs with identical preferences. The designed alignment resembles correlated preferences in real life. An exchange opportunity is salient for objects $b$ and $d$ and it is symmetric between agents.

Based on this environment, three different stochastic assignment mechanisms are compared in the experiment: the simultaneous version of RSD (SP-RSD), the sequential version of RSD (OSP-RSD), and top trading cycles with random initial endowments (TTC).

In this paper, I use standard theory and the behavioral theory of obvious strategy-proofness to test the institutional design of stochastic assignment mechanisms. The mechanisms are evaluated based on three criteria: Strategy-proofness, obvious strategy-proofness, and Pareto efficiency.

Strategy-proofness (SP) requires truthful preference revelation to be the weakly dominant strategy for every agent. In addition, the behavioral theory of obvious strategy-proofness (OSP) takes cognitive limitations into account: Recognizing an obviously dominant strategy does not require hypothetical thinking about the other agents' actions (Li, 2016). An assignment is Pareto-efficient if it is in the core, i.e., no coalition improvement is possible. Efficiency is defined for each group as the sum of earnings divided by the sum of earnings which would have been obtained under the Pareto-efficient assignment. 


\subsection{Simultaneous random serial dictatorship (SP-RSD)}

In the simultaneous version of RSD (SP-RSD), all agents submit the entire list of preferences. It works as follows (description from Chen and Sönmez, 2002).

- Agents submit a full list of preferences over objects.

- Nature draws an order of the agents from a uniform distribution.

- The first agent is assigned her top choice.

- The second agent is assigned her top choice among the remaining objects.

- The last agent is assigned the remaining object.

By regarding agents' preferences, SP-RSD dominates the random assignment of objects (Erdil, 2014). SP-RSD is strategy-proof and Pareto-efficient (Zhou, 1990; Abdulkadiroğlu and Sönmez, 1998). SP-RSD is not obviously strategy-proof, because recognizing the weakly dominant strategy involves identifying the other agents' potential actions by hypothetical thinking (Li, 2016).

\subsection{Sequential random serial dictatorship (OSP-RSD)}

In the sequential version of RSD (OSP-RSD), agents are taking turns and choose one object from a given set:

- Nature draws an order of the agents from a uniform distribution.

- The first agent chooses her top choice from the set of objects.

- The second agent chooses her top choice among the set of remaining objects.

- The last agent is assigned the remaining object.

Like SP-RSD, OSP-RSD is strategy-proof and Pareto-efficient (Zhou, 1990; Abdulkadiroğlu and Sönmez, 1998). Additionally, OSP-RSD is obviously strategyproof because picking the largest prize does not involve hypothetical reasoning about the actions of the other players (Li, 2016). 


\subsection{Top trading cycles with random endowments (TTC)}

Now I introduce initial endowments to the house allocation problem. Initial random endowments for Gale's top trading cycles algorithm induce a housing market. The housing market (Shapley and Scarf, 1974) is a quadruple $\langle I, O, \succ, \eta\rangle$, where $\eta$ is the initial endowment assignment added to the house allocation problem. TTC with strict preferences selects the unique core allocation of the housing market and coincides with the competitive equilibrium (Roth and Postlewaite, 1977). Therefore, this mechanism is also known as "core from random endowments" (Abdulkadiroğlu and Sönmez, 1998; Pathak, 2008). TTC with random endowments works as follows (description adapted from Abdulkadiroğlu and Sönmez, 1998).

- Agents submit a full list of preferences over objects.

- Nature draws an initial assignment from a uniform distribution.

- Step 1: Every agent points to the agent owning her most preferred object. Every object points to its owner. There is at least one cycle. A cycle is an order of agents $\{1,2\}$ where agent 1 points to agent 2 and agent 2 points to agent 1. Execute trades and remove cycles. Go to the next step if there are remaining agents.

- Step k: Every remaining agent points to the agent owning her most preferred object among the remaining objects. Objects point to their owners. Execute trades and remove cycles. Repeat until there are no remaining agents.

TTC obtaining the unique core allocation is strategy-proof (Roth, 1982) as well as individually rational, and Pareto-efficient (Ma, 1994). However, TTC is not obviously strategy-proof for markets with 3 or more agents because the opponent players' actions need to be taken into account to recognize the dominant strategy.

Proposition Li. (Li, 2016) TTC with $n \geq 3$ is not OSP-implementable.

TTC coincides with RSD if there are no existing endowments. TTC, SP-RSD, and OSP-RSD are strategy-proof and ex-post Pareto-efficient.

Theorem AS. (Abdulkadiroğlu and Sönmez, 1998) RSD is the same lottery mechanism as TTC with random endowments. 


\subsection{Pareto efficiency in the experiment}

TTC, SP-RSD, and OSP-RSD are ex-post Pareto-efficient in the experimental environment under the standard assumption of dominant strategy play.

Proposition 1. The core allocation under SP-RSD, OSP-RSD, and TTC is: One agent gets her first, two agents get their second, and one agent gets her third preference.

Sketch of proof. According to Abdulkadiroğlu and Sönmez (1998), TTC and serial dictatorships are ex-post Pareto-efficient. Therefore, TTC, SP-RSD, and OSP-RSD are ex-post Pareto-efficient and obtain the same outcome distribution. Independent of the order of the random queue, the following outcome distribution is obtained for the agents $I=\{1,2,3,4\}$.

\begin{tabular}{|c|c|c|}
\hline 1 & 2 & 34 \\
\hline$a$ & $a$ & $a \quad a$ \\
\hline & $b$ & $\begin{array}{ll}d & d\end{array}$ \\
\hline$c$ & $c$ & $c \quad c$ \\
\hline & $d$ & $b$ \\
\hline
\end{tabular}

The boxes around the preference profiles illustrate that only one agent gets her top choice object $a$, two agents receive their second-best objects $b$ and $d$, one agent receives her third preference $c$, and no agent receives her last preference. The outcome distribution of the core allocation from the mechanisms TTC, SP-RSD, and OSP-RSD, given the induced preference profiles $\succ_{i}$, is $1-2-1-0$.

Since the preference profiles are highly correlated in the experimental parametrization, there is little room for differences in efficiency resulting from non-dominant strategy play. 


\subsection{Predictions}

Since TTC, SP-RSD, and OSP-RSD are strategy-proof in standard game theory, subjects are predicted to play dominant strategies under all three mechanisms.

Hypothesis 1. (Dominant strategy play) $T T C=S P-R S D=O S P-R S D$.

Since OSP-RSD is obviously strategy-proof, subjects are predicted by the behavioral theory of OSP to play the dominant strategy more often under OSP-RSD than under SP-RSD or TTC if assuming limited ability for contingent reasoning. The amount of dominant strategy play is predicted by the following relation:

Hypothesis 2. (Behavioral theory) $S P-R S D<O S P-R S D \wedge T T C<O S P-R S D$.

Hypotheses 1 and 2 are competing hypotheses about dominant strategy play from standard and behavioral theory.

Since the mechanisms are ex-post Pareto-efficient, subjects are predicted to obtain the core allocation under all three mechanisms. Proposition 1 predicts that this core allocation assigns one agent her top choice, two agents their second-best choice, and one agent her third choice.

Hypothesis 3. (Pareto efficiency) $T T C=S P-R S D=O S P-R S D$. 


\section{Experimental design}

Figure 1 summarizes the experimental setup implementing a one-shot game under complete information. The three mechanisms, TTC, SP-RSD, and OSP-RSD, are compared between-subjects. In the beginning, participants are randomly divided into groups of 4 , where each participant is randomly assigned a role within each group. Roles correspond to preference profiles $\succ_{i}$ and remain constant. Then, subjects are informed about two mechanisms, one after another. Subjects only get to know mechanism 2 after they have completed mechanism 1. They are either confronted with the pair TTC/ SP-RSD or with the pair TTC/ OSP-RSD. Subjects exercise through each allocation rule and answer control questions. Instructions are available in the Appendix.

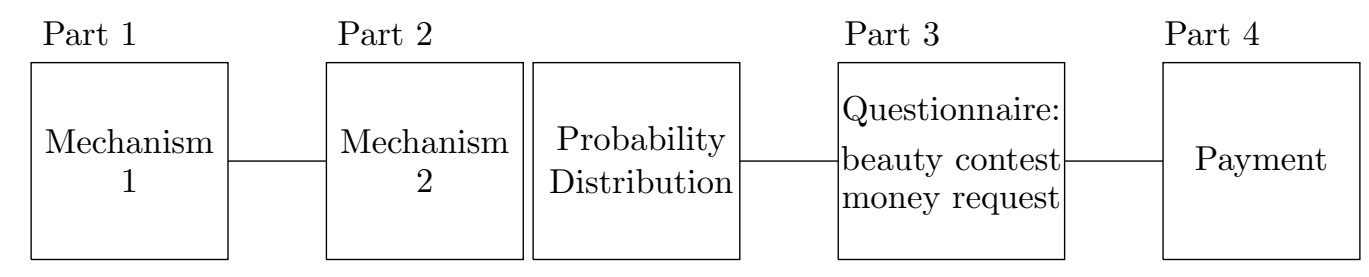

Figure 1: Experimental setup.

Part 1. Subjects state their preferences under mechanism 1. This one-shot decision is the main variable of interest for the between-group comparison. Mechanisms are either TTC, SP-RSD, or OSP-RSD. The mechanism is determined randomly. Subjects do not receive feedback until part 4. TTC and SP-RSD require submission of a list of preferences over 4 objects. In OSP-RSD, subjects select an object from a set of objects.

OSP-RSD is implemented using the strategy method (Selten, 1967): Subjects choose their preferred object 4 times from 4 sets of objects at the same time. The aim is to make the simultaneous submission of a list in TTC and SP-RSD comparable to selecting objects from sets in OSP-RSD. The reason is that listing 4 objects in an order may produce larger error rates than selecting a single object. Therefore, each subject is confronted with 4 sets of objects when making their choice in OSP-RSD. Subjects are ordered sequentially, but they do not know in which position of the sequence they are when they decide about the 4 sets. 
Figure 2 illustrates the implementation of OSP-RSD with an example. Subjects face 4 choices in 4 different sets containing $4,3,2$, and 1 object(s), without knowing their position in the sequence. Only one set of objects is the payoff-relevant set depending on the others' previous choice (corresponding to the actual position in the sequence). For instance, the subject at position 1 chooses her preferred object from sets $\{a, b, c, d\},\{b, c, d\},\{b, c\}$, and $\{b\}$, without knowing that she is at position 1 . Her payoff-relevant decision is about the object from $\{a, b, c, d\}$.

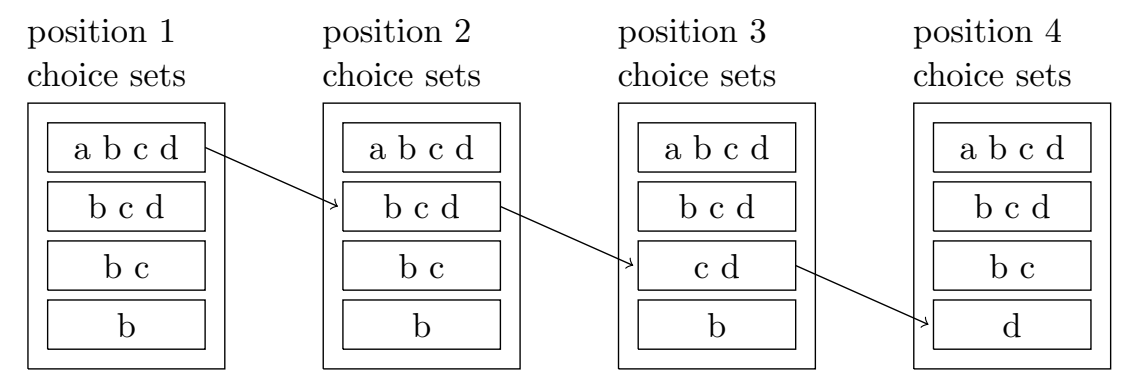

Figure 2: OSP-RSD: Every subject chooses from 4 choice sets without knowing her actual position in the sequence. The decision of the subject at position 1 in the first choice set impacts the second choice set of the agent at position 2, etc.

This method provides a fair comparison of dominant strategy play between submitting a preference list and choosing from sets of objects because the task of listing 4 objects can be regarded as choosing an object for each individual rank. Importantly, the 4 choices in OSP-RSD do not involve contingent reasoning about others' actions, but require picking the preferred object from each choice set.

The single choice according to the actual position in the random sequence, as used by Li (2016), can be reconstructed from this method by only considering the decision about choice set 1 by position 1 , the decision about choice set 2 by position 2, and so on. Henceforth, I refer to this reconstruction as OSP-RSD(1).

Part 2. In order to elicit a preference over mechanisms, subjects also make a choice under mechanism 2. Mechanism 2 is either SP-RSD or OSP-RSD if mechanism 1 was TTC, or it is TTC if mechanism 1 was SP-RSD or OSP-RSD. This order is randomized to control for order effects.

Only one mechanism (either 1 or 2) is paid out randomly in the end to avoid hedging possibilities. I elicit the preference for a mechanism by giving subjects the possibility to determine the probability distribution for the random payment. They 
do not know about the distributions until they finish mechanism 2. They choose between 50:50 and 80:20, respectively, for each mechanism. The latter options, implying $80 \%$ probability of the preferred mechanism to be paid out, cost ten euro cents. An indifferent subject would choose 50:50, whereas a subject with a strict preference would choose the option 80:20, respectively.

Part 3. The questionnaire contains two games to capture extreme forms of contingent reasoning. In the 2-person beauty contest game (Grosskopf and Nagel, 2008), two players form a group and submit an integer guess between 0 and 100. The closest guess to $2 / 3$ of the average of the group wins 2 euros. The weakly dominant strategy is stating 0 . I presuppose that stating 0 is a sufficient condition for perfect contingent reasoning about the hypothetical guess of the other person.

In contrast, the 11-20 money request game of Arad and Rubinstein (2012) does not have a pure strategy Nash equilibrium, but an intuitively salient level-0 strategy of requesting 20. In this game, two players form a group and submit an integer number between 11 and 20 points. They keep this amount. If one player submits exactly one integer less than the other player, then this player receives 20 points on top. The benefit of this second game is that answering 20 does not involve thinking about the others' hypothetical actions, and it is at the same time a sensible answer. Additionally, the psychological need for cognition scale (Cacioppo and Petty, 1982; Bless et al., 1994), risk attitude measurement (Holt and Laury, 2002), and demographic characteristics are included.

Part 4. After the experiment, the uncertainty is resolved by running the mechanisms. Subjects are paid out one mechanism with the probability determined in part 2. They receive 10 euros for their top choice, 7 euros for their second, 4 euros for their third, and 1 euro for their least preferred object. Payments are administered anonymously and privately.

Procedure. Sessions were conducted in March 2017 at the laboratory of the Technical University of Berlin. In sum, $N=228$ participants took part. They were on average 26 years old and $52 \%$ of them were female. The majority of subjects $(57 \%)$ were students in engineering, mathematics, or physics. Sessions lasted around 60 minutes. Average earnings (including show-up fee) were 16 euros (min: 9; $\max : 22$ ). The experiment was programmed using the software $z$-Tree (Fischbacher, 2007). Participants were recruited using ORSEE (Greiner, 2015). 


\section{Results}

\subsection{Dominant strategy play}

Do individuals play their dominant strategies? This question is analyzed based on the subjects' stated preferences in mechanism 1 (where they do not know about the second mechanism). Hypothesis 1 states that subjects play dominant strategies under all mechanisms. This paper does not find supporting evidence. The behavioral Hypothesis 2 states that subjects play dominant strategies more often in OSP-RSD than in TTC. This is supported by the data.

Result 1. (Dominant strategies: RSD versus TTC) On average, dominant strategies are played more often in RSD mechanisms than in TTC. Being in the TTC mechanism decreases the likelihood of submitting the dominant strategy by $30 \%$ compared to SP-RSD.

Support. Table 1 presents proportions of dominant strategy play under each mechanism. Subjects play dominant strategies significantly more often in the RSD mechanisms than in TTC. Table 4 presents probit estimation results of dominant strategy play. The marginal effect of TTC compared to SP-RSD (row 2) is significant and robust to controlling for additional explanatory factors in model (4).

Table 1: Proportion of dominant strategy play.

\begin{tabular}{lcccc}
\hline \hline Mechanism & Obs. & $\begin{array}{c}\text { Dominant } \\
\text { strategies }\end{array}$ & \multicolumn{2}{c}{ Mann-Whitney U test p-value } \\
\cline { 4 - 5 } & & $53.3 \%$ & & \\
\hline TTC & 23 & $86.1 \%$ & 0.0002 & \\
SP-RSD & 18 & $95.3 \%$ & $<0.0001$ & 0.029 \\
OSP-RSD(1) & 16 & $78.1 \%$ & 0.0021 & 0.813 \\
OSP-RSD(4) & 16 & & vs. SPD \\
\hline
\end{tabular}

The average of each group is one independent observation. Reported $p$-values are based on one-tailed testing, except for SP-RSD vs. TTC. For TTC and SP-RSD, dominant strategy play is defined as submitting the full preference list truthfully. For OSP-RSD(1), dominant strategy play is defined as choosing the largest prize according to the position in the queue. For $\operatorname{OSP}-\mathrm{RSD}(4)$, it is defined as choosing each time the largest prize out of 4 different sets with $4,3,2$, and 1 alternative(s). 
The behavioral Hypothesis 2 also states that subjects play dominant strategies more often in OSP-RSD than in SP-RSD. Employing two different definitions of OSP-RSD, labeled OSP-RSD(1) and OSP-RSD(4), this paper finds mixed evidence.

Both OSP-RSD definitions are obtained from the same choice data and method. OSP-RSD(1) is nested in OSP-RSD(4). In OSP-RSD(1) - consistent with the definition in Li (2016) - participants play a dominant strategy if they choose the largest prize according to their actual position in the random queue. In OSP-RSD(4), dominant strategy play is defined as choosing the largest prize in each and every set of objects simultaneously. Here, subjects make 4 choices from 4 different sets containing 4, 3, 2, and 1 object(s).

Result 2. (Dominant strategies: OSP-RSD versus SP-RSD) On average, subjects more often play their dominant strategies in OSP-RSD(1) (95\%) than in SP-RSD (86\%). There is no significant difference between $O S P-R S D(4)$ and $S P-R S D$.

Support. Table 1 presents proportions of dominant strategy play in each mechanism. Dominant strategy play in $\operatorname{OSP}-\operatorname{RSD}(1)$ occurs significantly more often than in SP-RSD. Dominant strategies are not played significantly more frequently in OSP-RSD(4) than in SP-RSD.

Result 2 indicates that the difference between SP-RSD and OSP-RSD depends on the definition of dominant strategy play in OSP-RSD. The OSP-RSD(1) finding replicates previous results from $\mathrm{Li}$ (2016). Here, dominant strategy play is based on one single choice from one set of objects. However, the difference between OSP-RSD and SP-RSD vanishes when 4 choices are considered in OSP-RSD(4).

Why then is the frequency of dominant strategy-play lower in $\operatorname{OSP}-\operatorname{RSD}(4)$ compared to OSP-RSD(1)? The reason is that the misrepresentation or error rate is amplified across multiple choice sets. In OSP-RSD(4), 5/14 cases of non-dominant strategy play can be attributed to selecting the second-best object from the full choice set containing 4 objects and at the same time playing the dominant strategy in the choice set containing 3 objects. Another $7 / 14$ cases can be attributed to selecting the second-best object in the choice set containing 3 objects, while playing the dominant strategy in the full choice set with 4 objects. 


\subsection{Misrepresentation strategies}

Which misrepresentation strategies are mainly used? Figure 3 presents an overview of the manipulation strategies in SP-RSD and TTC. ${ }^{3}$ Preference lists from mechanism 1 are considered only (one-shot, between-subjects).

The most common manipulation strategy is to put the 2nd preference on top of the list. This second-best on top strategy accounts for 26/43 cases of non-dominant strategy play in TTC, for 3/10 in SP-RSD, for 3/3 in OSP-RSD(1), and for 12/14 cases of non-dominant strategy play in $\mathrm{OSP}-\mathrm{RSD}(4)$.

Figure 3 illustrates the tendency that the second-best on top strategy is played more frequently in TTC than in SP-RSD. This indicates that it is easier to recognize for subjects in SP-RSD that they cannot outsmart the mechanism by putting their second-best on top of the list.

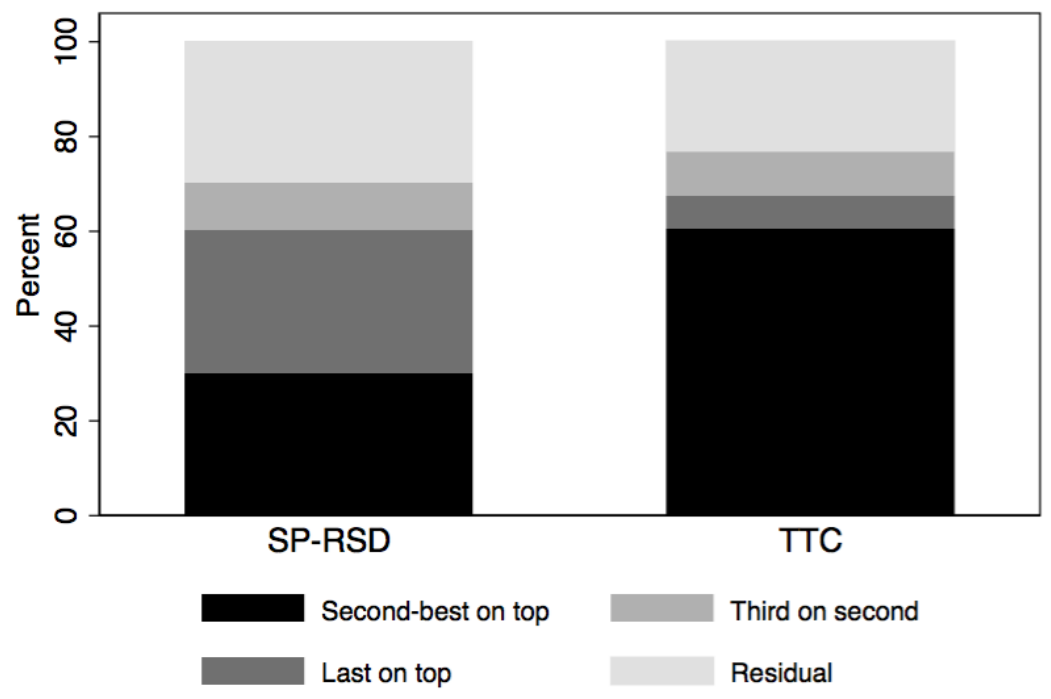

Figure 3: Misrepresentation strategies in SP-RSD and TTC.

The other two strategies of putting the last preference on top of the list and of putting the third preference on the second position are less frequent.

\footnotetext{
${ }^{3}$ In OSP-RSD(1), all three cases of manipulation are second-best on top. In the experiment, four subjects use more than one of the three manipulation strategies. Then, the first strategy is taken into account according to the order: second on top - last on top - third on second.
} 


\subsection{Efficiency}

Does not playing the dominant strategy impact the efficiency of the outcome? Efficiency is defined for each group as the sum of payoffs divided by the sum of Pareto-efficient earnings. Observed efficiency is based on one random queue. Expected efficiency is based on simulations with 10.000 random queues. Hypothesis 3 states that all mechanisms result in the Pareto-efficient assignment. This is not supported by the evidence.

Result 3. (Pareto-efficient assignments) In expectation, SP-RSD attains the Pareto-efficient assignment $30 \%$ more often than TTC.

Support. Table 2 presents in the last column the proportions of assignments ending in the Pareto-efficient welfare level using the simulated expected efficiency. The expected proportion of Pareto-efficient assignments is significantly different between SP-RSD (72.2\%) and TTC (39.1\%), according to the Mann-Whitney U test $(N=41, p=0.037$, two-tailed).

Table 2: Efficiency.

\begin{tabular}{lccc}
\hline \hline Mechanism & Observed & \multicolumn{2}{c}{ Expected efficiency } \\
\cline { 3 - 4 } & efficiency & Mean from & Proportion of Pareto- \\
mean & 10.000 random draws & efficient assignments \\
\hline TTC & 0.953 & $0.943(0.016)$ & $39.1 \%$ \\
SP-RSD & 0.976 & $0.964(0.018)$ & $72.2 \%$ \\
OSP-RSD $(1)$ & 0.960 & - & - \\
\hline Random lists & - & $0.821(0.026)$ & - \\
\hline
\end{tabular}

The Pareto-efficient welfare level under dominant strategy play is 1. Standard errors in parenthesis. Observed efficiency is based on one random queue. Expected efficiency is based on simulations with 10.000 random queues.

OSP-RSD(1) cannot be evaluated based on expected efficiency. Based on observed efficiency, the proportion of Pareto-efficient assignments in OSP-RSD(1) is 81.3\%. This indicates that more dominant strategy play leads to more groups attaining the Pareto-efficient welfare level. Due to the correlated experimental environment, the mean efficiency does not differ between mechanisms (see Section 2.4). 


\subsection{Mechanisms}

Why do individuals not play dominant strategies? I explore potential explanations including the subjects' ability for contingent reasoning, procedural preferences for the mechanisms, and risk aversion.

Ability for contingent reasoning. The ability for contingent reasoning is captured with data from two additional games. I focus on identifying the extremes. Perfect contingent reasoning is defined as playing the dominant strategy in the 2-person beauty contest game by choosing 0 . Non-contingent reasoning is defined as playing the level-0 strategy in the 11-20 money request game by requesting $20 .{ }^{4}$ Perfect as well as no ability for contingent reasoning result in the same behavior.

Result 4. (Ability for contingent reasoning) Individuals with a perfect ability for contingent reasoning, as well as individuals with no ability for contingent reasoning, play dominant strategies. The marginal effect of non-contingent reasoning on dominant strategy play is $14 \%$.

Support. Table 3 presents cases of dominant strategy play by the subjects' ability for contingent reasoning. Subjects ex-ante classified as having an ability, as well as subjects classified as having no ability for contingent reasoning, play dominant strategies. Table 4 presents probit estimation results of dominant strategy play. Non-contingent (row 7) is significantly positively related to playing the dominant strategy. The marginal effect of non-contingent, compared to those not requesting 20 in the 11-20 money request game, is obtained from model (3).

Table 3: Ability for contingent reasoning and dominant strategy play.

\begin{tabular}{llrr}
\hline \hline Ability & Definition & Dominant strategies & Total cases \\
\hline Contingent reasoning & Beauty contest guess $=0$ & 15 & 15 \\
Intermediate level & $\begin{array}{l}\text { Beauty contest guess }>0 \wedge \\
\text { Money request }<20\end{array}$ & 138 & 192 \\
Non-contingent reasoning & Money request $=20$ & 20 & 22 \\
\hline Total & & 172 & 228 \\
\hline
\end{tabular}

\footnotetext{
${ }^{4}$ Sample distributions of guesses and requests can be found in the Appendix. Both sets are disjunct except for 1 subject guessing 0 and requesting 20 at the same time.
} 
Result 4 indicates that both extreme expressions of the ability for contingent reasoning result in dominant strategy play. This means that intermediate levels of the ability for contingent reasoning matter for dominant strategy play.

Table 4: Probit regression of dominant strategy play.

\begin{tabular}{|c|c|c|c|c|c|c|}
\hline & & & \multicolumn{4}{|c|}{ Predicted variable: Dominant strategy play } \\
\hline & & & (1) & $(2)$ & (3) & (4) \\
\hline 1 & $\begin{array}{l}\text { Mechanisms: } \\
\text { (Reference: SP-RSD) }\end{array}$ & OSP-RSD $(1)$ & $\begin{array}{l}0.142^{*} \\
(0.080)\end{array}$ & $\begin{array}{c}0.082 \\
(0.091)\end{array}$ & $\begin{array}{c}0.087 \\
(0.086)\end{array}$ & $\begin{array}{c}0.081 \\
(0.092)\end{array}$ \\
\hline 2 & & TTC & $\begin{array}{c}-0.293^{* * *} \\
(0.071)\end{array}$ & $\begin{array}{c}-0.304^{* * *} \\
(0.068)\end{array}$ & $\begin{array}{c}-0.304^{* * *} \\
(0.070)\end{array}$ & $\begin{array}{c}-0.295^{* * *} \\
(0.065)\end{array}$ \\
\hline 3 & $\begin{array}{l}\text { Preference for: } \\
\text { (Reference: Indifferent) }\end{array}$ & SP-RSD & & $\begin{array}{c}-0.267^{* * *} \\
(0.089)\end{array}$ & $\begin{array}{c}-0.246^{* * *} \\
(0.089)\end{array}$ & $\begin{array}{c}-0.253^{* * *} \\
(0.088)\end{array}$ \\
\hline 4 & & OSP-RSD & & $\begin{array}{l}0.118^{*} \\
(0.070)\end{array}$ & $\begin{array}{l}0.119^{*} \\
(0.071)\end{array}$ & $\begin{array}{c}0.123 \\
(0.081)\end{array}$ \\
\hline 5 & & TTC & & $\begin{array}{c}0.040 \\
(0.067)\end{array}$ & $\begin{array}{c}0.027 \\
(0.069)\end{array}$ & $\begin{array}{c}0.005 \\
(0.067)\end{array}$ \\
\hline 6 & Beauty contest guess & & & & $\begin{array}{l}-0.002^{*} \\
(0.001)\end{array}$ & $\begin{array}{l}-0.002^{*} \\
(0.001)\end{array}$ \\
\hline 7 & Non-contingent & & & & $\begin{array}{l}0.137^{* *} \\
(0.064)\end{array}$ & $\begin{array}{l}0.240^{* *} \\
(0.108)\end{array}$ \\
\hline 8 & Risk aversion & & & & $\begin{array}{l}-0.001 \\
(0.014)\end{array}$ & $\begin{array}{c}0.007 \\
(0.015)\end{array}$ \\
\hline 9 & $\begin{array}{l}\text { Non-contingent } \times \\
\text { Risk aversion }\end{array}$ & & & & & $\begin{array}{l}-0.043 \\
(0.068)\end{array}$ \\
\hline 10 & Set of controls ${ }^{+}$ & & No & No & No & Yes \\
\hline & Clusters & & 57 & 57 & 57 & 57 \\
\hline & $\mathrm{N}$ & & 228 & 228 & 228 & 228 \\
\hline
\end{tabular}

Predicted variable: 1 if dominant strategy play and 0 otherwise. Data from mechanism 1. Average marginal effects reported. Standard errors clustered at group level in parentheses. Non-contingent: 1 if request is 20 in the 11-20 money request game and 0 otherwise.

+ Set of controls: demographics, player role, field of study, math grade, need for cognition.

Significance levels: ${ }^{*} p<0.10,{ }^{* *} p<0.05,{ }^{* * *} p<0.01$.

Table 4 also shows that the level of contingent reasoning, approximated by the beauty contest guess (row 6), is related to dominant strategy play. Note that the definition of perfect contingent reasoning cannot be included in the probit model because it perfectly predicts dominant strategy play. ${ }^{5}$

\footnotetext{
${ }^{5}$ Contingent reasoning is defined as playing the weakly dominant strategy in the 2-person
} 
Procedural preferences. Do subjects have a preference for mechanisms? Overall, around $40 \%$ of the subjects strictly prefer one assignment mechanism to the other, while the majority of participants is indifferent between the mechanisms. The TTC mechanism is preferred by $18 \%$, SP-RSD by another $18 \%$, and OSP-RSD by $22 \%$ of the subjects. This indicates that a substantial fraction of subjects has a preference for an assignment mechanism. Moreover, the preference for SP-RSD is found to be related to dominant strategy play.

Result 5. (Procedural preferences) The likelihood of playing a dominant strategy is $25 \%$ lower for individuals having a preference for SP-RSD compared to indifference.

Support. Table 4 presents probit estimation results of dominant strategy play. Having a preference for SP-RSD (row 3) is associated with a significantly decreased likelihood of dominant strategy play compared to the reference group of being indifferent. The marginal effect from the full model (4) is reported.

Result 5 suggests that having a procedural preference for SP-RSD is negatively related to dominant strategy play. More specifically, this effect can be partly attributed to subjects preferring the SP-RSD mechanism and not playing the dominant strategy in TTC. In 12/15 of the overall cases of having a preference for SP-RSD and not playing the dominant strategy, subjects do not play the dominant strategy in TTC.

Risk attitudes. Table 4 indicates that there is no significant relation at the $5 \%$ significance level between risk attitudes (row 8) and dominant strategy play. Risk aversion interacted with non-contingent (row 9) is not significantly related to dominant strategy play either. This indicates that the relation between not engaging in hypothetical thinking as measured by non-contingent (row 7) and dominant strategy play cannot merely be explained by subjects being risk-averse.

beauty contest game by choosing 0 . However, choosing 0 in the beauty contest game perfectly predicts dominant strategy play in the mechanisms. This yields complete separability and the failure to fit the estimation models in Table 4 by maximum likelihood. 


\section{Discussion and conclusion}

In this paper, I test the performance of RSD and TTC for the house allocation problem without endowments and find that - in contrast to standard theory - RSD outperforms TTC. Dominant strategies are played more frequently and the Paretoefficient welfare level is attained more often in RSD. This result stands in contrast to previous findings of Chen and Sönmez $(2002,2004)$ on the house allocation problem with existing endowments. Their opposite finding that TTC outperforms RSD mainly results from subjects choosing the option not to participate in RSD because they could be worse off than their endowment matching. In line with the theory of Abdulkadiroğlu and Sönmez (1998), this option is not available in the present problem without endowments since everyone participates.

In the experiment, I introduce a new method to compare simultaneous with sequential mechanisms. I assume that listing objects in a preference order involves a decision about each individual rank in the list. Therefore, submitting a list (in TTC and SP-RSD) is more comparable to choosing from multiple choice sets than from a single choice set as in Li (2016). Sequential OSP-RSD is implemented as subjects choosing their preferred object from 4 choice sets without knowing their position in the sequence. This method has a second virtue: The single choice according to the actual position in the sequence can be reconstructed from this data. The single choice from one set is nested in the 4 choices from 4 sets. The new method comes at a cost: The decision situation of choosing simultaneously from different sets is cognitively more complex than choosing from one set. Nevertheless, reconstructing the single choice, I replicate the finding of $\mathrm{Li}(2016)$ that $2.6 \%$ (my experiment: $4.7 \%$ ) do not play dominant strategies in OSP-RSD.

I do not find a difference in dominant strategy play between the sequential OSPRSD and the simultaneous SP-RSD when using the data from the new method. The reason is that the error rate is higher when choosing from multiple sets: Subjects play the dominant strategy by choosing the highest prize in the first choice set, but fail to do so in the second choice set and the other way around.

A substantial fraction of subjects of $40 \%$ prefers one assignment mechanism over the other, while the majority is indifferent. This evidence is in line with the previous finding that preferences over mechanisms which yield identical expected outcomes can differ systematically (Schmelzer, 2016). Further, the preference for SP-RSD is 
negatively related to dominant strategy play. This is driven by individuals who do not play the dominant strategy in TTC, indicating a preference for RSD.

I provide a first approach to identify extreme forms of contingent reasoning. Individuals classified as having a perfect ability, as well as subjects with no ability for contingent reasoning, play dominant strategies. This implies that the behavior of individuals with an intermediate level of contingent reasoning is crucial for the performance of mechanisms. For intermediate levels, mechanisms become key if the dominant strategy is easier to see than in others. In a related paper, Basteck and Mantovani (2016) find that subjects with a low cognitive ability play the dominant strategy less frequently in the deferred acceptance algorithm than high-ability subjects. The relation between the individuals' ability for contingent reasoning and their cognitive ability in matching markets remains an open question.

Several explanations for preference misrepresentation are discussed in the matching literature (see Hassidim et al., 2017). In my experiment, the most prevalent form of preference misrepresentation is to place the second-best object at the top of the list; even more frequently in TTC than in SP-RSD. The top-choice object is identical for all subjects in a group while the second-best is only identical for half of the group. This misrepresentation behavior is consistent with the self-selection explanation of Chen and Pereyra (2016) that subjects rank an object lower if the perceived chance of receiving it is very low.

The findings of my paper can inform the behavioral theory on obvious strategyproofness (OSP). Strategic complexity of TTC and RSD seem to play a role, but in a different way than predicted by the behavioral theory of OSP. While OSP predicts the result that OSP-RSD outperforms TTC, it cannot explain the result that SPRSD outperforms TTC. SP-RSD and TTC may differ in their complexity in a way so far not captured by OSP. A refinement of contingent reasoning might be helpful. The number or the quality of the contingencies may play a role in explaining why the frequency of dominant strategy play is larger in SP-RSD than in TTC. TTC not only involve, contingent reasoning about the random priorities, as SP-RSD does, but additionally about the mutually beneficial exchange opportunities given the initial random assignment.

From a policy perspective, the planners' choice matters since the strategyproofness of the one mechanism is easier to understand than the strategy-proofness 
of the other. Given the experimental evidence, it is easier for individuals to recognize that they cannot game the system by misrepresenting their preferences in RSD than in TTC. Therefore, the RSD mechanism may be considered instead of the TTC mechanism for assignment problems in which individuals' preferences are correlated and in which the individuals do not have pre-existing claims for the objects to be assigned.

In conclusion, TTC and RSD are not equivalent as predicted by standard theory. In the absence of existing endowments, RSD yields more dominant strategy play than TTC. Dominant strategy play is related to the ability of individuals for contingent reasoning. Individuals with extremely high and low levels of contingent reasoning play dominant strategies. This can inform market design in practice: Strategy-proof and optimal assignment mechanisms may not yield the predicted results if individuals are boundedly rational. What is more, one strategy-proof market design may perform better with real people than the other. 


\section{References}

AbdulkadiroĞLu, A. and Sönmez, T. (1998). Random serial dictatorship and the core from random endowments in house allocation problems. Econometrica, 66 (3), 689-701.

— and Sönmez, T. (1999). House allocation with existing tenants. Journal of Economic Theory, 88 (2), 233-260.

Arad, A. and Rubinstein, A. (2012). The 11-20 money request game: A level-k reasoning study. American Economic Review, 102 (7), 3561-73.

Ashlagi, I. and Gonczarowski, Y. A. (2015). No stable matching mechanism is obviously strategy-proof. mimeo.

Basteck, C. and Mantovani, M. (2016). Cognitive ability and games of school choice. mimeo.

Bless, H., Wänke, M., Bohner, G., Fellhauer, R. F. and Schwarz, N. (1994). Need for cognition: eine Skala zur Erfassung von Engagement und Freude bei Denkaufgaben. Zeitschrift für Sozialpsychologie, 25.

Cacioppo, J. T. and Petty, R. E. (1982). The need for cognition. Journal of Personality and Social Psychology, 42 (1), 116.

Charness, G. and Levin, D. (2009). The origin of the winner's curse: A laboratory study. American Economic Journal: Microeconomics, 1 (1), 207-236.

Chen, L. and Pereyra, J. S. (2016). Self-selection in school choice. mimeo.

Chen, Y. and Sönmez, T. (2002). Improving efficiency of on-campus housing: An experimental study. American Economic Review, 92 (5), 1669-1686.

— and Sönmez, T. (2004). An experimental study of house allocation mechanisms. Economics Letters, 83 (1), 137-140.

ErDil, A. (2014). Strategy-proof stochastic assignment. Journal of Economic Theory, 151, 146-162.

Esponda, I. and VesPA, E. (2014). Hypothetical thinking and information extraction in the laboratory. American Economic Journal: Microeconomics, 6 (4), 180-202.

— and - (2016). Contingent preferences and the sure-thing principle: Revisiting classic anomalies in the laboratory. mimeo.

FischBACHeR, U. (2007). z-Tree: Zurich toolbox for ready-made economic experiments. Experimental Economics, 10 (2), 171-178. 
GreineR, B. (2015). Subject pool recruitment procedures: organizing experiments with ORSEE. Journal of the Economic Science Association, 1 (1), 114-125.

Grosskopf, B. and Nagel, R. (2008). The two-person beauty contest. Games and Economic Behavior, 62 (1), 93-99.

Guillen, P. and Hakimov, R. (2016). Not quite the best response: Truth-telling, strategy-proof matching, and the manipulation of others. Experimental Economics, pp. $1-17$.

Hassidim, A., Marciano, D., Romm, A., Shorrer, R. I. et al. (2017). The mechanism is truthful, why aren't you? American Economic Review, 107 (5), 220-24.

-, Romm, A. and Shorrer, R. I. (2016). 'Strategic' Behavior in a strategy-proof environment. mimeo.

Holt, C. A. and Laury, S. K. (2002). Risk aversion and incentive effects. American Economic Review, 92 (5), 1644-1655.

Hylland, A. and Zeckhauser, R. (1979). The efficient allocation of individuals to positions. Journal of Political Economy, 87 (2), 293-314.

LI, S. (2016). Obviously strategy-proof mechanisms. mimeo.

MA, J. (1994). Strategy-proofness and the strict core in a market with indivisibilities. International Journal of Game Theory, 23 (1), 75-83.

Moraga, J. F.-H. and RApoport, H. (2014). Tradable immigration quotas. Journal of Public Economics, 115, 94-108.

Ngangoue, K. and Weizsäcker, G. (2015). Learning from unrealized versus realized prices. mimeo.

Olson, M. and Porter, D. (1994). An experimental examination into the design of decentralized methods to solve the assignment problem with and without money. Economic Theory, 4 (1), 11-40.

Pathak, P. A. (2008). Lotteries in student assignment: The equivalence of queueing and a market-based approach. mimeo.

Pycia, M. and Troyan, P. (2016). Obvious dominance and random priority. mimeo.

ReEs-Jones, A. (2017). Suboptimal behavior in strategy-proof mechanisms: Evidence from the residency match. Games and Economic Behavior, in press.

Roth, A. E. (1982). Incentive compatibility in a market with indivisible goods. Economics letters, 9 (2), 127-132. 
- and Postlewaite, A. (1977). Weak versus strong domination in a market with indivisible goods. Journal of Mathematical Economics, 4 (2), 131-137.

Schmelzer, A. (2016). Single versus multiple randomization in matching mechanisms. mimeo.

Selten, R. (1967). Die Strategiemethode zur Erforschung des eingeschränkt rationalen Verhaltens im Rahmen eines Oligopolexperiments. In H. Sauermann (ed.), Beiträge zur Experimentellen Wirtschaftsforschung, Tübingen: J.C.B. Mohr (Paul Siebeck), pp. $136-168$.

Shapley, L. and ScARF, H. (1974). On cores and indivisibility. Journal of Mathematical Economics, 1 (1), 23-37.

Troyan, P. (2016). Obviously strategyproof implementation of allocation mechanisms. mimeo.

ZHANG, J. (2016). Level-k reasoning in school choice. mimeo.

Zhang, L. and Levin, D. (2017). Bounded rationality and robust mechanism design: An axiomatic approach. American Economic Review, 107 (5), 235-239.

Zhou, L. (1990). On a conjecture by Gale about one-sided matching problems. Journal of Economic Theory, 52 (1), 123-135. 


\section{Appendix for online publication}

\section{Figures}

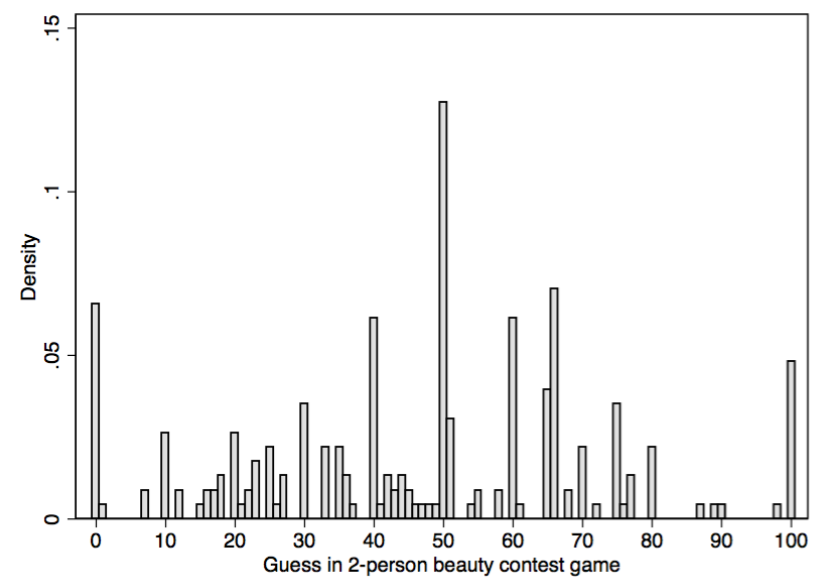

Figure 4: Distribution of guesses in the 2-person beauty contest game.

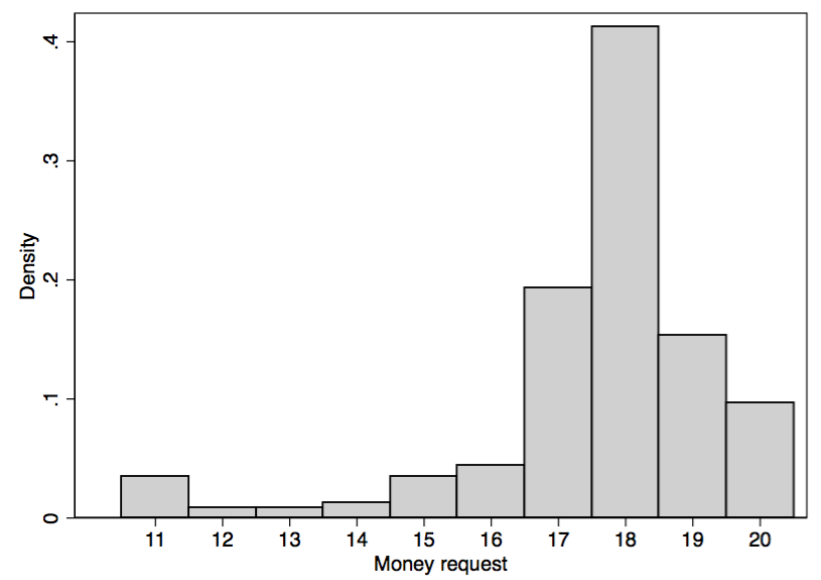

Figure 5: Distribution of requests in the 11-20 money request game. 


\section{Instructions}

Welcome! You are about to take part in an economic study in decision-making. You will receive a show-up fee of 6 euros. Additionally, you will be able to earn a substantial amount of money. It is therefore crucial that you read these explanations carefully. The present instructions are identical for all participants.

Please switch off your mobile phone and do not communicate with other participants. If you have any questions, please raise your hand. We will then come over to you. Any violation of these rules means you will be excluded from the experiment and from any payments.

During the experiment, we will calculate in points. The total number of points you earn in the course of the experiment will be transferred into euro at the end, at a rate of

$$
1 \text { euro }=20 \text { points. }
$$

The procedure and payment details are described below.

At the beginning of the experiment, all participants are randomly divided into groups of four. You will not get to know the identity of the other participants in your group. You stay in the same group during the experiment.

In the experiment, we simulate procedures that assign positions to applicants. A central clearinghouse takes care of the assignment procedure. You and the other participants are applicants. Within each group, you are randomly assigned the role of an applicant. This role remains the same throughout the experiment.

The following payment table determines your payoff at the end of the experiment.

Payment table.

\begin{tabular}{rcccc}
\hline Points & $\begin{array}{c}\text { Applicant } \\
\text { green }\end{array}$ & $\begin{array}{c}\text { Applicant } \\
\text { blue }\end{array}$ & $\begin{array}{c}\text { Applicant } \\
\text { red }\end{array}$ & $\begin{array}{c}\text { Applicant } \\
\text { yellow }\end{array}$ \\
\hline 200 points & $\mathrm{W}$ & $\mathrm{W}$ & $\mathrm{W}$ & $\mathrm{W}$ \\
140 points & $\mathrm{X}$ & $\mathrm{X}$ & $\mathrm{Z}$ & $\mathrm{Z}$ \\
80 points & $\mathrm{Y}$ & $\mathrm{Y}$ & $\mathrm{Y}$ & $\mathrm{Y}$ \\
20 points & $\mathrm{Z}$ & $\mathrm{Z}$ & $\mathrm{X}$ & $\mathrm{X}$ \\
\hline
\end{tabular}

In this payment table, you can see how many points each applicant receives for each assigned position. This table is equivalent in both tasks. For instance, if applicant green is assigned position $\mathrm{W}$ in the allocation procedure, then he receives 200 points. 
If applicant green is assigned position $\mathrm{X}$, then he receives 140 points; for position $\mathrm{Y}$ he receives 80 points and for position $\mathrm{Z}$ he receives 20 points.

\section{Procedure}

- Task 1

- Task 2

- Questionnaire

- Payment

You will receive more detailed information about task 1 and task 2 on your computer screen after the experiment starts. In each task, we simulate a procedure that assigns positions to applicants.

One of both tasks (either 1 or 2) is randomly determined and paid out to you in the end. The precise probability for the random payment will be determined in the experiment.

only for TTC and SP-RSD:

$<<\underline{\text { Your Decision }}$

In each task, you will make a decision about a ranking of positions. You will receive details on the use of this ranking in the procedure during the experiment. All four applicants submit a ranking. You may submit any ranking. All positions have to be listed. Rank 1 means the top rank, rank two the second-highest, rank 3 the third-highest, and rank 4 the lowest rank. $>>$

Do you have any questions? If this is the case, then please raise your hand. We will answer your questions individually. Thank you for participating in this experiment! 


\section{Instructions for SP-RSD}

All applicants submit one ranking of positions. Then the procedure works as follows.

- List of applicants: A fair lottery determines a list of applicants. This means each applicant has an equal chance of becoming first, second, third, or fourth on this list.

- The first applicant on the list of applicants receives the position at rank 1 of his submitted ranking.

- The second applicant on the list of applicants receives the position with the highest rank among the remaining positions of his submitted ranking.

- The third applicant on the list of applicants receives the position with the highest rank among the remaining positions of his submitted ranking.

- The fourth applicant on the list of applicants is assigned to the remaining position.

\section{An example}

Consider for illustration purposes the following example. There are three applicants (gray, black, and white) and three positions (A, B, and C).

- Rankings. Assume that the applicants submitted the following rankings:

$$
\begin{array}{llll}
\text { Applicant gray: } & \operatorname{rank} 1=\mathrm{B} & \operatorname{rank} 2=\mathrm{C} & \operatorname{rank} 3=\mathrm{A} . \\
\text { Applicant black: } & \operatorname{rank} 1=\mathrm{C} & \operatorname{rank} 2=\mathrm{A} & \operatorname{rank} 3=\mathrm{B} . \\
\text { Applicant white: } & \operatorname{rank} 1=\mathrm{B} & \operatorname{rank} 2=\mathrm{C} & \operatorname{rank} 3=\mathrm{A} .
\end{array}
$$

Important: These sample rankings are chosen arbitrarily and only serve illustrational purposes. They provide no guidance for your decision-making in the experiment!

- List of applicants. Assume the following list of applicants:

$$
\text { black - gray - white }
$$

Please complete the following sentences.

- The first applicant on the list of applicants receives the position at rank 1 of his submitted ranking. That is, applicant black receives position

- The second applicant on the list of applicants receives the position with the highest rank among the remaining positions (A and B) of his submitted ranking. That is, applicant gray receives position

- The third applicant on the list of applicants is assigned to the remaining position. That is, applicant white receives position 


\section{Instructions for OSP-RSD}

The procedure works as follows.

- List of applicants: A fair lottery determines a list of applicants. This means each applicant has an equal chance of becoming first, second, third, or fourth on this list.

- Set of positions: Available positions which have not yet been chosen.

- The first applicant on the list of applicants chooses one position from the full set of positions.

- The second applicant on the list of applicants chooses one position from the remaining positions in the set of positions.

- The third applicant on the list of applicants chooses one position from the remaining positions in the set of positions.

- The fourth applicant on the list of applicants receives to the remaining position.

\section{An example}

Consider for illustration purposes the following example. There are three applicants (gray, black, and white) and three positions (A, B, and C).

- List of applicants. Assume the following list of applicants:

$$
\text { black - gray - white }
$$

- Assume, for instance, that applicant black prefers position A and applicant gray prefers position B.

Please complete the following sentences.

- The first applicant on the list of applicants chooses one position from the full set of positions (A, B, C). That is, applicant black chooses position

- The second applicant on the list of applicants chooses one position from the remaining positions in the set of positions $(\mathrm{B}, \mathrm{C})$. That is, applicant gray chooses position

- The third applicant on the list of applicants receives to the remaining position. That is, applicant white receives

\section{$\underline{\text { Your Decision }}$}

In this task, you will see 4 different sets of positions corresponding to a place on the list of applicants. One set of positions is relevant for your final payment.

Only at the end of the experiment will you learn which set of positions is relevant for your payment and with that, which place on the list of applicants you have.

Your decision is to choose your preferred position out of each of the 4 sets of positions. 


\section{Instructions for TTC}

All applicants submit one ranking of positions. Then the procedure works as follows.

- Tentative assignment: Each applicant is first tentatively assigned to one position based on a fair lottery. This means each applicant has an equal chance to be assigned to a particular position. This assignment is tentative.

- Next, the rankings are used to determine mutually beneficial exchanges between two or more participants.

- Queue: In order to perform mutually beneficial exchanges, a queue is determined by a fair lottery. The lottery determines each applicant's place in the queue. Each queue is equally likely. This means that each applicant has an equal probability of becoming first, second, ..., or last in the queue.

- The specific allocation process is explained below. It starts with the first applicant in the queue. The application of the first applicant in the queue is submitted to the position with rank 1 on his ranking.

- If the application is submitted to his tentatively assigned position, then his tentative assignment is finalized, i.e., he receives the position. The applicant and his assignment are removed from subsequent allocations. The process continues with the next applicant in line.

- If the application is submitted to another position, say position $\mathrm{S}$, then the first applicant in the queue who is tentatively assigned position $\mathrm{S}$ is moved to the top of the queue directly in front of the requester.

- Whenever the queue is modified, the process continues similarly: An application is submitted to the highest ranked position for the applicant at the top of the queue.

- A mutually beneficial exchange is obtained when a cycle of applications are made in sequence, which benefits all affected applicants, e.g., A applies to B's tentatively assigned position, and B applies to A's tentatively assigned position. In this case, the exchange is completed and the applicants as well as their assignments are removed from subsequent allocations.

- The process continues until all applicants are assigned a position.

\section{An example}

Consider for illustration purposes the following example. There are three applicants (gray, black, and white) and three positions (A, B, and C). 
- Rankings. Assume that the applicants submitted the following rankings:

$$
\begin{array}{llll}
\text { Applicant gray: } & \operatorname{rank} 1=\mathrm{B} & \operatorname{rank} 2=\mathrm{C} & \operatorname{rank} 3=\mathrm{A} . \\
\text { Applicant black: } & \operatorname{rank} 1=\mathrm{C} & \operatorname{rank} 2=\mathrm{A} & \operatorname{rank} 3=\mathrm{B} . \\
\text { Applicant white: } & \operatorname{rank} 1=\mathrm{B} & \operatorname{rank} 2=\mathrm{C} & \operatorname{rank} 3=\mathrm{A} .
\end{array}
$$

Important: These sample rankings are chosen arbitrarily and only serve illustrational purposes. They provide no guidance for your decision-making in the experiment!

- Tentative assignment. Assume the following tentative assignment of positions:

\begin{tabular}{|c|c|c|}
\hline Applicant gray & Applicant black & Applicant white \\
\hline C & B & A \\
\hline
\end{tabular}

- Queue. Assume the following queue of applicants:

$$
\text { gray - black - white }
$$

Please complete the following sentences.

- The application of the first applicant in the queue is submitted to the position with rank 1 on his ranking. That is, the application of applicant gray is submitted to position

- This application is not submitted to his tentatively assigned position C. The queue is modified: The first applicant in the queue who is tentatively assigned position $\mathrm{B}$ is moved to the top of the queue directly in front of the requester. At the top of the queue is now applicant

- The queue is modified:

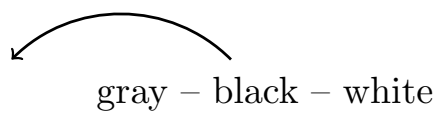

The new queue is:

$$
\text { black - gray - white }
$$

- The application of the (new) first applicant in the queue is submitted to the position with rank 1 on his ranking. That is, the application of applicant black is submitted to position 
- This application of applicant black is not submitted to his tentatively assigned position B. Therefore, the queue is modified: The first applicant in the queue who is tentatively assigned position $\mathrm{C}$ is moved to the top of the queue directly in front of the requester. At the top of the queue is now applicant

- The queue is modified:

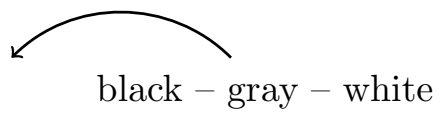

The new queue is:

$$
\text { gray - black - white }
$$

- Now a cycle of applications is made in sequence and with that a mutually-beneficial exchange is obtained. The following two applicants exchange their tentatively assigned positions and are removed with their assignments from subsequent allocations:

Applicant and

Applicant

- Illustration of the exchange of positions:

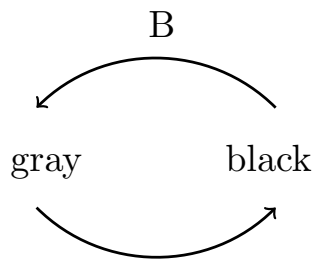

C

- Applicant white is the remaining applicant in the assignment process. His application is submitted to the remaining position:

- Since applicant white has already been tentatively assigned to this position, the assignment is finalized and he gets the position. Applicant white and his position are removed from the allocation process.

- The final assignment is:

\begin{tabular}{|c|c|c|}
\hline Applicant gray & Applicant black & Applicant white \\
\hline$B$ & $C$ & $A$ \\
\hline
\end{tabular}

